\title{
Sodium-Glucose Co-Transporter-2 (SGLT2) Inhibitors: Comparing Trial and Real World Use (Study Protocol)
}

\author{
Andrew McGovern • Michael Feher • Neil Munro • \\ Simon de Lusignan
}

Received: December 5, 2016/ Published online: January 30, 2017

(C) The Author(s) 2017. This article is published with open access at Springerlink.com

\begin{abstract}
Background: Sodium-glucose co-transporter-2 (SGLT2) inhibitors (gliflozins) are the newest class of medication available to treat type 2 diabetes (T2DM). Recent findings from the first complete cardiovascular safety trial in SGLT2 inhibitors, the Empagliflozin, Cardiovascular Outcomes, and Mortality in type 2 diabetes (EMPA-REG OUTCOMES) trial, demonstrated reduced cardiovascular outcomes in people with high cardiovascular risk. How to apply these findings to clinical practice remains unclear, with questions remaining on who will reap this cardiovascular benefit.

Aim: To describe the proportion of people in the real world currently treated with SGLT2 inhibitors who meet the inclusion criteria of the

Enhanced content To view enhanced content for this article go to http://www.medengine.com/Redeem/ 2587F0603F89AAF4.
\end{abstract}

A. McGovern $(\bowtie) \cdot$ N. Munro $\cdot$ S. de Lusignan Department of Clinical and Experimental Medicine, University of Surrey, Guildford, UK

e-mail: a.mcgovern@surrey.ac.uk

M. Feher

Beta Cell Centre for Diabetes, Chelsea and

Westminster Hospital, 369 Fulham Road, London, UK

M. Feher

Warwick Medical School, Warwick University, Coventry, UK
EMPA-REG trial and therefore could expect the cardiovascular benefit identified by the trial. Similarly, to describe the proportion of people from the whole T2DM population who could also expect this same benefit.

Design and Setting: Routinely collected data from UK primary care in the Royal College of General Practitioners (RCGP) Research and Surveillance Centre (RSC) database will be used. The study population will include all people with T2DM within this database (approximately 60,000). We will perform a cross-sectional investigation to describe the characteristics of people currently using SGTL2 inhibitors compared with the population of the EMPA-REG trail. We will similarly compare the characteristics of the RCGP RSC T2DM cohort with the inclusion criteria of the EMPA-REG trial.

Method: People with T2DM using a pre-existing verified clinical ontological process will be identified, as will people with prescriptions for SGLT2 inhibitors and other medications using Read coded and other proprietary coding systems. Descriptive statistics will be used to characterise the key clinical characteristics of people with T2DM using SGLT2 inhibitors and to compare these characteristics to people included in EMPA-REG trial; the proportion of people who match the trial criteria will be reported.

Planned Outputs: Peer review publication reporting the real world lessons for clinical practice. 
Funding: AstraZeneca.

Keywords: EMPA-REG; SGLT2 inhibitor; Real world evidence; Type 2 diabetes

\section{INTRODUCTION}

Sodium glucose co-transporter 2 (SGLT2) inhibitors, also known as gliflozins, are a new class of oral medication for the treatment of hyperglycaemia in type 2 diabetes (T2DM). They improve glycaemic control through the inhibition of glucose reuptake by SGLT2 in the proximal tubule of the kidney $[1,2]$. This action is independent of insulin secretion and activity and, therefore, these agents are not considered to predispose to hypoglycaemia [3]; they also promote modest, but sustained, weight loss [4]. Inhibition of SGLT2 also promotes urinary sodium loss $[5,6]$ which, along with weight reduction, may be responsible for the early blood pressure-lowering effects observed in trials [7]. The reduction in weight and blood pressure seen in clinical trials are likely to be clinically meaningful and are potentially useful additional benefits of these agents.

Three SGLT2 inhibitors are available in both Europe and the USA, namely, canagliflozin, dapagliflozin and empagliflozin. SGLT2 inhibitors have been found to provide excellent glycaemic improvement in randomised controlled trials when used as a single agent [8] and when used in combination with metformin $[3,4,9]$, sulfonylureas $[10,11]$ or insulin $[12,13]$. However, clinical trials are performed on selected patient groups and, therefore, the trial results may not be fully replicated in 'real world' clinical practice. In this context, The Association of British Clinical Diabetologists nationwide exenatide audit demonstrated a differing real world efficacy profile to that reported by clinical trials [14]. Whilst early data with dapagliflozin (the first drug available in the SGLT2 inhibitor class) has demonstrated good efficacy in the real world [15] additional analyses on a larger scale are still needed.

Recent findings from the first cardiovascular safety trial in SGLT2 inhibitors to be completed, the BI 10773 (Empagliflozin) Cardiovascular
Outcome Event Trial in Type 2 Diabetes Mellitus Patients (EMPA-REG OUTCOME) trial demonstrated reduced cardiovascular outcomes in people with high cardiovascular risk (Table 1) who were treated with empagliflozin [16]. How these findings can be extrapolated into real world clinical practice remains unclear. Other cardiovascular safety trials are still ongoing and will help to answer some of these questions as their inclusion criteria are somewhat broader than those of the EMPA-REG trial, with a lower cardiovascular risk population included. In the meantime, a comparison of the characteristics of people treated with SGLT2 inhibitors in clinical practice with those of the EMPA-REG trial is important to enable an understanding of how these important trial results can be applied to clinical practice. A measure of the proportion of people with T2DM, to whom these trial criteria apply in clinical practice, is vitally important to enable correct interpretation of the results.

\section{AIM AND METHODS}

The study will be a cross-sectional analysis of all people with T2DM included in the Royal College of General Practitioners Research (RCGP) and Surveillance Centre (RSC) database to identify people initiated on SGLT2 inhibitors and to describe their cardiovascular risk profile. The proportion of people who have a similar cardiovascular risk profile to those included in the EMPA-REG trial will also be reported.

\section{OBJECTIVES}

The aim of this study will be to compare the clinical characteristics of people initiated on SGLT2 inhibitors with those of people included in the EMPA-REG OUTCOMES trial.

\section{Primary Objectives}

1. To identify how many people initiated on an SGLT2 inhibitor in clinical practice meet the inclusion criteria for the EMP-REG trial.

2. To provide a breakdown of this proportion by: 
Table 1 A summary of the major cardiovascular safety trials in sodium glucose co-transporter 2 inhibitors

\begin{tabular}{|c|c|c|c|}
\hline Medication & $\begin{array}{l}\text { Cardiovascular } \\
\text { safety trial }\end{array}$ & Trial characteristics & Trial outcomes \\
\hline Canagliflozin & CANVAS [27] & $\begin{array}{l}\text { vs. placebo }(n=4330) \\
\text { Inclusion criteria: age } \geq 50 \text { years, high } \\
\text { CV risk or age } \geq 30 \text { years, with } \\
\text { previous MI } \\
\text { Follow-up: up to } 7 \text { years } \\
\text { Primary outcome: time to composite } \\
\text { of CV death, nonfatal MI or } \\
\text { ischaemic stroke }\end{array}$ & Study completion: February 2017 \\
\hline Dapagliflozin & $\begin{array}{l}\text { DECLARE-TIMI } \\
58 \text { [28] }\end{array}$ & $\begin{array}{l}\text { vs. placebo }(n=17,276) \\
\text { Inclusion criteria: age } \geq 40 \text { years, high } \\
\text { CV risk } \\
\text { Follow-up: up to } 6 \text { years } \\
\text { Primary outcome: time to composite } \\
\text { of CV death, MI or ischaemic stroke }\end{array}$ & Study completion: April 2019 \\
\hline Empagliflozin & $\begin{array}{l}\text { EMPA-REG } \\
\text { OUTCOME } \\
{[16]}\end{array}$ & $\begin{array}{l}\text { vs. placebo }(n=7034) \\
\text { Inclusion criteria: age } \geq 18 \text { years, very } \\
\text { high } C V \text { risk } \\
\text { Follow-up: } 3.1 \text { years } \\
\text { Primary outcome: time to composite } \\
\text { of CV death, nonfatal MI or stroke }\end{array}$ & $\begin{array}{l}\text { Reduction in the primary outcome compared } \\
\text { with placebo (HR 0.86; 95\% CI 0.74-0.99; } \\
\quad P=0.04) \\
\text { Reduced hospitalisation for heart failure (HR } \\
0.65 ; 95 \% \text { CI } 0.57-0.82 ; P<0.001 \text { ) }\end{array}$ \\
\hline
\end{tabular}

$C V$ cardiovascular, $C I$ confidence interval, $H R$ hazard ratio, $M I$ myocardial infarction

(a) the number of people with each inclusion criteria for EMPA-REG,

(b) by duration of diabetes,

(c) by number of concurrent diabetes agents and presence or absence of insulin use.

3. To describe the clinical characteristics (age, gender distribution, weight, blood pressure, renal function and time since diagnosis) of people in the each of the above groups.

\section{Secondary Objectives}

4. To also identify how many people initiated on each SGLT2 inhibitor separately (canagliflozin, dapagliflozin, and empagliflozin) meet the inclusion criteria for the EMPA-REG trial, comparing each SGLT2 inhibitor as a subgroup analysis-providing sufficient numbers are available.

5. To identify how many people in clinical practice, in the entire cohort, meet the inclusion criteria for the EMP-REG trial.

6. To provide a breakdown of this proportion by:

(a) the number of people with each inclusion criteria for EMPA-REG,

(b) by duration of diabetes,

(c) by number of concurrent diabetes agents and presence or absence of insulin use.

7. To describe the clinical characteristics (age, gender distribution, weight, blood pressure, 
renal function and time since diagnosis) of people in the each of the above groups.

\section{DATA SOURCE}

Routinely collected English general practice data will be used to perform the study. These data are suitable for this type of analysis for several reasons [17]. Firstly, English general practice is a registration-based system-people have to register with a single general practitioner (GP), and all individuals have a unique national patient identifier, namely, the National Health Service (NHS) number, which facilitates linking data on patient movement from one general practice to another as well as deaths. This number makes the population denominator reliable and valid. The NHS number also helps facilitate data linkage, pathology results for example, to the correct record. Secondly, many GPs computerised in the 1990s, with most prescribing being carried out using computerised records. Coding of chronic disease data and laboratory links become nearly universal from around 2004. Although not widely used internationally, the UK uses the Read code system, an extensive coding system which allows the detailed coding of diagnosis, symptoms, signs, investigations, therapy and health service management [18]. Diabetes data are particularly well recorded, although care is needed to accurately find cases and to differentiate between the different ways data are recorded on the different computerised medical record systems used by GPs [19, 20]. Sufficient details on prescription data are available to facilitate the study of the use and persistence of medicine in real world therapy [21].

The RCGP RSC database includes the primary care records from 128 primary care practices distributed across England $(1.7 \%$ of all practices) and provides a broadly representative population sample [22]. All included data are recorded using the Read code 5-byte version 2 coding hierarchy. The coded data include comprehensive diagnosis and treatment information, prescriptions and laboratory data. Inclusion of data recording UK primary care pay-for-performance targets have led to a high level of data completeness in these records, particularly in the population of people with T2DM [23].

We will use data from all the included primary care practices collated after January 1, 2016 and will include all patients with a diagnosis of T2DM who are older than 18 years on or before this date. In those with T2DM we will identify all those people initiated on SGLT2 inhibitors (canagliflozin, dapagliflozin, or empagliflozin) at any time before January 1, 2016. For those with T2DM and a prescription for SGLT2 inhibitor we will report their clinical characteristics and the proportion of people with a cardiovascular risk similar to that of the EMPA-REG trial population.

\section{DATA ANALYSIS}

Our aim is to define and make explicit our approach to using real world data to create real world evidence $[24,25]$. To this end, we use a two-stage informatics ontology-based process to identify people with T2DM [21]. This is a concept-based approach to case and outcome identification [26]. In brief, the two stages are the identification of all people with diabetes (stage 1) and then categorisation by diabetes type (stage 2 ). People with diabetes are identified using one or more of the following: (1) a diabetes diagnosis code, (2) glucose and glycated hemoglobin (HbA1c) test results (two or more consistent with diabetes) or (3) the use of diabetes therapies (excluding metformin). People are classified by diabetes type based on their medication usage history, diabetes type-specific diagnosis codes and other key clinical characteristics (these include age at diagnosis, duration of oral medication use, and body mass index at diagnosis).

The high cardiovascular risk inclusion criteria for the EMPA-REG study are given in Table 2. We will identify people with each of these cardiovascular risk factors using our ontological process to identify the nearest matching clinical diagnostic codes, or other codes which identified the presence of the risk factor. We will include the full description of this process and the final list of codes generated in the final manuscript. 
Table 2 A comparison of the inclusion criteria of the EMPA-REG OUTCOMES trial with the nearest match available from routine UK primary care data

\section{EMPA-REG OUTCOMES trial inclusion criteria Nearest match using routinely collected primary care data}

History of myocardial infarction

History of myocardial infarction $>2$ months prior to informed consent

Coronary artery disease

Evidence of multi-vessel coronary artery disease, i.e. in $\geq 2$ major coronary arteries or the left main coronary artery, documented by any of the following:

Presence of significant stenosis: $\geq 50 \%$ luminal narrowing during angiography (coronary or multi-slice computed tomography)

Previous revascularisation (percutaneous transluminal coronary angioplasty \pm stent or coronary artery bypass graft $>2$ months prior to consent

The combination of revascularisation in one major coronary artery and significant stenosis $(\geq 50 \%$ luminal narrowing) in another major coronary artery

Evidence of single-vessel coronary artery disease, $\geq 50 \%$ luminal narrowing during angiography (coronary or multi-slice computed tomography) not subsequently successfully re-vascularised, with at least 1 of the following: A positive non-invasive stress test for ischaemia

Hospital discharge for unstable angina $\leq 12$ months prior to consent

Unstable angina

Unstable angina $>2$ months prior to consent with evidence of single- or multi-vessel coronary artery disease

History of stroke

History of stroke (ischaemic or haemorrhagic) $>2$ months prior to consent
Coding of a myocardial infarction or other definite indicator of a myocardial infarction

Evidence of coronary artery disease documented by any of the following:

Coding of double coronary artery disease

Previous revascularisation (percutaneous transluminal coronary angioplasty \pm stent or coronary artery bypass graft) of the coronary arteries

NB. Coding of the precise percutaneous intervention performed in primary care is limited. Usually only a high level code is included to indicate the procedure has been undertaken. This coronary artery disease code search is therefore likely to slightly overestimate the number of people who meet the trial criteria

Coding of unstable angina or code which indicates poor angina control

Coding of stroke (ischemic or haemorrhagic) 
Table 2 continued

\begin{tabular}{|c|c|}
\hline EMPA-REG OUTCOMES trial inclusion criteria & $\begin{array}{l}\text { Nearest match using routinely collected } \\
\text { primary care data }\end{array}$ \\
\hline \multicolumn{2}{|l|}{ Peripheral artery disease } \\
\hline $\begin{array}{l}\text { Occlusive peripheral artery disease documented by any of the } \\
\text { following: }\end{array}$ & $\begin{array}{l}\text { Coding of peripheral artery disease documented by any } \\
\text { of the following: }\end{array}$ \\
\hline Limb angioplasty, stenting or bypass surgery & Limb angioplasty, stenting or bypass surgery \\
\hline Limb or foot amputation due to circulatory insufficiency & Limb or foot amputation \\
\hline $\begin{array}{l}\text { Evidence of significant peripheral artery stenosis }(>50 \% \text { on } \\
\text { angiography, or }>50 \% \text { or haemodynamically significant } \\
\text { via non-invasive methods) in } 1 \mathrm{limb}\end{array}$ & $\begin{array}{l}\text { Coding of peripheral arterial disease of the lower } \\
\operatorname{limb}(s) \text { including claudication, and peripheral } \\
\text { gangrene }\end{array}$ \\
\hline Ankle brachial index $<0.9$ in $\geq 1$ ankle & A recorded ankle brachial index $<0.9$ \\
\hline
\end{tabular}

EMPA-REG OUTCOMES BI 10773 (Empagliflozin) Cardiovascular Outcome Event Trial in Type 2 Diabetes Mellitus Patients

Where specific disease information is not available from routine data (either due to coding limitations or due to limitations in non-specific data entry) we have used broader criteria, for example, percentage luminal narrowing is rarely recorded in primary care; we therefore include all people with coded coronary artery stenosis

\section{STATISTICAL METHODS}

Standard descriptive statistics (mean, standard deviations etc.) will be used to describe the characteristics of the various populations. This will include describing baseline HbA1c at initiation of therapy with SGLT2 inhibitor. We will report the crude rates of each outcome measure for each cohort, as well as the proportion of people with each outcome of interest together with $95 \%$ confidence intervals.

\section{STRENGTHS AND LIMITATIONS}

The strengths of the dataset have been broadly eluded to in the "Data Source" section. The large-scale ( $>1.2$ million records) of this very complete real world evidence dataset is a particular strength. Despite the large size of the dataset only a minor of people with T2DM have been initiated on SGLT2 inhibitors to date and therefore subgroup analyses may not have sufficient power to resolve differences between groups.

An additional limitation is the potential for missing data on EMPA-REG inclusion criteria in the primary care record. A number of patients may have the condition of interest without documentation of this in the primary care record. However, as all the EMPA-REG inclusion criteria are major cardiovascular risk factors and the monitoring and recording of these risk factors are part of current primary care pay for performance targets in the UK (Quality and Outcome Framework), this effect is likely to be small. Any additional limitations identified during the conduct of the study will be discussed in the final study manuscript.

\section{COMPLIANCE WITH ETHICS GUIDELINES}

All data to be used have been anonymised at the point of data extraction. No clinically identifiable information will be made available to researchers or in any publications. The study has been tested against the Health Research Authority (HRA)/Medical Research Council (MRC) "is this research" tool (http://www.hradecisiontools.org.uk/research/) and is considered to be an audit of current practice when compared to best available evidence. The study 
therefore does not require specific ethical approval. Approval for this work has been granted by the RCGP RSC study approval committee.

\section{CONCLUSION}

This real world evidence cross-sectional analysis will demonstrate which proportion of people with T2DM, in an unselected population, are likely to benefit from the cardiovascular protection demonstrated in the EMPA-REG trial. These data will provide clinicians with valuable insights into the best applications for these important trial data.

\section{ACKNOWLEDGEMENTS}

This publication was supported by a grant from AstraZeneca, including funding for publication charges. The authors would like to acknowledge project manager Filipa Ferreira and SQL programmer Rachel Byford for their respective contributions. This study is considered to be an audit of current practice when tested against the Health Research Authority (HRA)/Medical Research Council (MRC) "is this research" tool and therefore did not require specific ethical approval. Approval for use of the RCGP RSC data to undertake this work was granted by the RCGP RSC study approval committee. All authors had full access to all of the data in this study and take complete responsibility for the integrity of the data and accuracy of the data analysis. All named authors meet the International Committee of Medical Journal Editors (ICMJE) criteria for authorship for this manuscript, take responsibility for the integrity of the work as a whole and have given final approval for the version to be published.

Author contributions. Andrew McGovern conceived the idea. Andrew McGovern and Simon de Lusignan drafted the manuscript. Michael Feher and Neil Munro reviewed the manuscript and study design and contributed to the final manuscript draft.
Disclosures. Andrew McGovern receives research funding from Eli-Lilly and AstraZeneca. Simon de Lusignan receives research funding from Eli-Lilly, GlaxoSmithKline, Takeda and AstraZeneca. Neil Munro and Michael Feher receive financial support for research, speaker meetings and consultancy from MSD, Merck, BMS, AstraZeneca, Pfizer, Novo, Eli-Lilly and Sanofi-Aventis.

Open Access. This article is distributed under the terms of the Creative Commons Attribution-NonCommercial 4.0 International License (http://creativecommons.org/licenses/ by-nc/4.0/), which permits any noncommercial use, distribution, and reproduction in any medium, provided you give appropriate credit to the original author(s) and the source, provide a link to the Creative Commons license, and indicate if changes were made.

\section{REFERENCES}

1. Fujita Y, Inagaki N. Renal sodium glucose cotransporter 2 inhibitors as a novel therapeutic approach to treatment of type 2 diabetes: clinical data and mechanism of action. J Diabetes Investig. 2014;5(3):265-75 (Epub 2014/05/21).

2. Rahmoune H, Thompson PW, Ward JM, Smith CD, Hong G, Brown J. Glucose transporters in human renal proximal tubular cells isolated from the urine of patients with non-insulin-dependent diabetes. Diabetes. 2005;54(12):3427-34 (Epub 2005/11/25).

3. Bailey CJ, Gross JL, Hennicken D, Iqbal N, Mansfield TA, List JF. Dapagliflozin add-on to metformin in type 2 diabetes inadequately controlled with metformin: a randomized, double-blind, placebo-controlled 102-week trial. BMC Med. 2013;11:43 (Epub 2013/02/22).

4. Strojek K, Yoon KH, Hruba V, Sugg J, Langkilde AM, Parikh S. Dapagliflozin added to glimepiride in patients with type 2 diabetes mellitus sustains glycemic control and weight loss over 48 weeks: a randomized, double-blind, parallel-group, placebo-controlled trial. Diabetes Ther. 2014;5(1):267-83 (Epub 2014/06/13).

5. Komoroski B, Vachharajani N, Feng Y, Li L, Kornhauser D, Pfister M. Dapagliflozin, a novel, selective SGLT2 inhibitor, improved glycemic control over 2 weeks in patients with type 2 diabetes mellitus. 
Clin Pharmacol Ther. 2009;85(5):513-9 (Epub 2009/01/09).

6. Tahrani AA, Barnett AH. Dapagliflozin: a sodium glucose cotransporter 2 inhibitor in development for type 2 diabetes. Diabetes Therapy. 2010;1(2):45-56.

7. Ptaszynska A, Hardy E, Johnsson E, Parikh S, List J. Effects of dapagliflozin on cardiovascular risk factors. Postgrad Med. 2013;125(3):181-9 (Epub 2013/06/12).

8. Ferrannini E, Ramos SJ, Salsali A, Tang W, List JF. Dapagliflozin monotherapy in type 2 diabetic patients with inadequate glycemic control by diet and exercise: a randomized, double-blind, placebo-controlled, phase 3 trial. Diabetes Care. 2010;33(10):2217-24 (Epub 2010/06/23).

9. Bailey CJ, Gross JL, Pieters A, Bastien A, List JF. Effect of dapagliflozin in patients with type 2 diabetes who have inadequate glycaemic control with metformin: a randomised, double-blind, placebo-controlled trial. Lancet. 2010;375(9733):2223-33 (Epub 2010/07/09).

10. Nauck MA, Del Prato S, Meier JJ, Duran-Garcia S, Rohwedder $\mathrm{K}$, Elze $\mathrm{M}$, et al. Dapagliflozin versus glipizide as add-on therapy in patients with type 2 diabetes who have inadequate glycemic control with metformin: a randomized, 52-week, double-blind, active-controlled noninferiority trial. Diabetes Care. 2011;34(9):2015-22 (Epub 2011/08/ 06).

11. Strojek K, Yoon KH, Hruba V, Elze M, Langkilde AM, Parikh S. Effect of dapagliflozin in patients with type 2 diabetes who have inadequate glycaemic control with glimepiride: a randomized, 24-week, double-blind, placebo-controlled trial. Diabetes Obes Metab. 2011;13(10):928-38 (Epub 2011/06/16).

12. Wilding JP, Norwood P, T'Joen C, Bastien A, List JF, Fiedorek FT. A study of dapagliflozin in patients with type 2 diabetes receiving high doses of insulin plus insulin sensitizers: applicability of a novel insulin-independent treatment. Diabetes Care. 2009;32(9):1656-62 (Epub 2009/06/17).

13. Wilding JP, Woo V, Soler NG, Pahor A, Sugg J, Rohwedder K, et al. Long-term efficacy of dapagliflozin in patients with type 2 diabetes mellitus receiving high doses of insulin: a randomized trial. Ann Intern Med. 2012;156(6):405-15 (Epub 2012/03/21).

14. Thong KY, Jose B, Sukumar N, Cull ML, Mills AP, Sathyapalan T, et al. Safety, efficacy and tolerability of exenatide in combination with insulin in the Association of British Clinical Diabetologists nationwide exenatide audit. Diabetes Obes Metab. 2011;13(8):703-10 (Epub 2011/03/18).

15. McGovern AP, Dutta N, Munro N, Watters K, Feher M. Dapagliflozin: clinical practice compared with pre-registration trial data. Br J Diabetes Vasc Dis. 2014;14(4):138-43.

16. Zinman B, Wanner C, Lachin JM, Fitchett D, Bluhmki E, Hantel S, et al. Empagliflozin, cardiovascular outcomes, and mortality in type 2 diabetes. N Engl J Med. 2015;373(22):2117-28.

17. de Lusignan S, van Weel C. The use of routinely collected computer data for research in primary care: opportunities and challenges. Fam Pract. 2006;23(2):253-63 (Epub 2005/12/22).

18. de Lusignan S. Codes, classifications, terminologies and nomenclatures: definition, development and application in practice. Inform Prim Care. 2005;13(1):65-70 (Epub 2005/06/14).

19. de Lusignan S, Sadek N, Mulnier H, Tahir A, Russell-Jones D, Khunti K. Miscoding, misclassification and misdiagnosis of diabetes in primary care. Diabet Med: J Br Diabet Assoc. 2012;29(2):181-9 (Epub 2011/09/03).

20. de Lusignan S, Liaw ST, Dedman D, Khunti K, Sadek $\mathrm{K}$, Jones $\mathrm{S}$. An algorithm to improve diagnostic accuracy in diabetes in computerised problem orientated medical records (POMR) compared with an established algorithm developed in episode orientated records (EOMR). J Innov Health Inform. 2015;22(2):255-64 (Epub 2015/08/08).

21. McGovern A, Hinton W, Correa A, Munro N, Whyte $M$, de Lusignan S. Real-world evidence studies into treatment adherence, thresholds for intervention and disparities in treatment in people with type 2 diabetes in the UK. BMJ Open. 2016;6(11):e012801 (Epub 2016/11/26).

22. Fleming DM, Miles J. The representativeness of sentinel practice networks. J Public Health (Oxf, Engl). 2010;32(1):90-6 (Epub 2009/09/18).

23. Correa A, Hinton W, McGovern A, van Vlymen J, Yonova I, Jones S, et al. Royal College of General Practitioners Research and Surveillance Centre (RCGP RSC) sentinel network: a cohort profile. BMJ Open. 2016;6(4):e011092 (Epub 2016/04/22).

24. de Lusignan S, Crawford L, Munro N. Creating and using real-world evidence to answer questions about clinical effectiveness. J Innov Health Inform. 2015;22(3):368-73 (Epub 2015/11/19).

25. McGovern A, Hinchliffe R, Munro N, de Lusignan S. Basing approvals of drugs for type 2 diabetes on real world outcomes. BMJ. 2015;351:h5829. 
26. Liaw ST, Taggart J, Yu H, de Lusignan S, Kuziemsky C, Hayen A. Integrating electronic health record information to support integrated care: practical application of ontologies to improve the accuracy of diabetes disease registers. J Biomed Inform. 2014;52:364-72 (Epub 2014/08/05).

27. Janssen Research \& Development LLC. CANVASCANagliflozin cardioVascular Assessment Study (CANVAS). ClinicalTrials.gov; 2009. https://
clinicaltrials.gov/ct2/show/NCT01032629. Accessed 14 Aug 2016.

28. AstraZeneca. Multicenter Trial to Evaluate the Effect of Dapagliflozin on the Incidence of Cardiovascular Events (DECLARE-TIMI58). ClinicalTrials.gov; 2012. https://clinicaltrials.gov/ct2/show/ NCT01730534. Accessed 14 Aug 2016. 